\title{
LE CHÂTEAU DE SAINT-GOBAIN ET LES CHÂTEAUX DE PLAN CONCENTRIQUE EN EUROPE OCCIDENTALE
}

PAR

\author{
PIERRE HÉLIOT
}

POUR nous le château de Saint-Gobain dans le Laonnais ${ }^{1}$ n'est presque plus qu'un souvenir. Bâti par les sires de Coucy, probablement dans la première moitié du $\mathrm{XIII}^{\mathrm{c}}$ siècle, transmis par héritage aux Luxembourg, il subit le contrecoup de la disgrâce sensationnelle d'un de ses possesseurs: le connétable de Saint-Pol. Louis XI le fit en effet démolir en 1475. En 1692 la manufacture royale de glaces, acquéreuse du terrain, établit ses ateliers à l'emplacement des ruines préalablement rasées. La célébrité qu'acquit dans la suite le nom de Saint-Gobain n'est pas due à ses seigneurs, mais au développement spectaculaire d'une société industrielle. De la forteresse il ne reste plus aujourd'hui que les salles et les galeries souterraines (Fig. 1). Pour le reste on a fait état de quelques dessins fort médiocres à tous égards et dont la valeur documentaire est des plus contestables. Exécutés bien après la destruction de l'édifice, ils interprètent peut-être, mais en ce cas avec une fidélité fort incertaine, une ou plusieurs images disparues du $\mathrm{XVI}^{\mathrm{e}}$ siècle ou du $\mathrm{XVII}^{\mathrm{e}} 2$.

Le château couronnait une croupe rocheuse. Son plan et ses dimensions approximatives nous sont suggérés par la principale des galeries: voie périphérique, creusée dans le sol et dont on a réussi à explorer la majeure partie. On a tout lieu de croire qu'elle accomplissait le circuit complet du monument. Son tracé semble nous révéler une bâtisse presque carrée, mesurant hors oeuvre 80 à $90 \mathrm{~m}$. de côté ${ }^{3}$, mais dont les faces, orientées chacune vers l'un des points cardinaux, eussent été construites en retrait de quatre bastions arrondis ou fortes tours d'angle, qui auraient atteint quelque $32 \mathrm{~m}$. de diamètre. Egal en ampleur aux précédents, un cinquième bastion regardait vers le midi et jouxtait celui

1 Aisne, canton de la Fère.

${ }^{2}$ Voir sur le château G. Dumas: St.-Gobain avant la manufacture établie en 1692, dans Fédération des soc. d'bist. et d'archéol. de l'Aisne: mémoires, XIV, 1968, pp. 33-38; I. PeYchìs: Notes sur le château-fort de St.-Gobain, Dijon, 1945. Peychès a publié les dessins dont il existe à la Bibliothèque nationale (Estampes, collect. Fleury, Ve 140 , t. XV, ff. 31 et 38 ) des variantes du XIX e siècle: un dessin à la plume et deux lithographies.

${ }^{3}$ D'après le plan publié par Peychès et reproduit ici-même, mais Peychès a évalué la longueur des côtés à une centaine de mètres. 


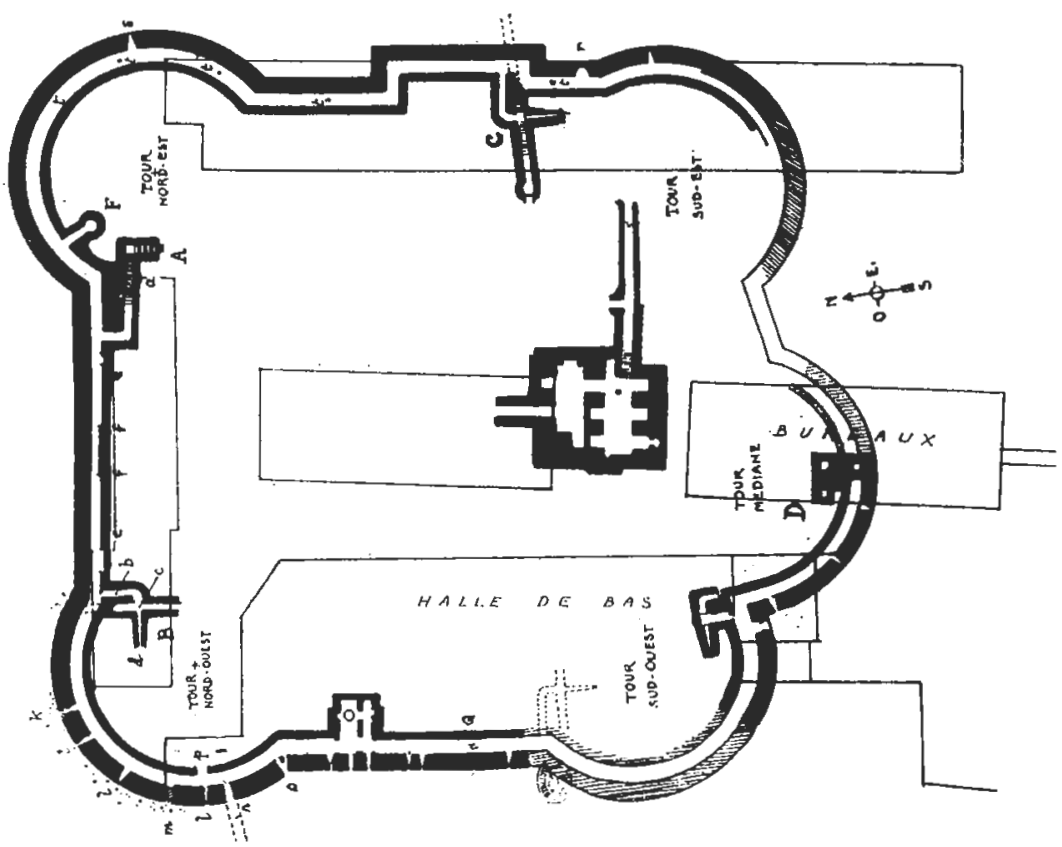

Fig. 1.-Saint-Gobain, Château, plan des galeries souterraines et des caves d'après I. Peychès.

du sud-ouest. Nous voici donc conduits à imaginer quatre courtínes, dessinant un carré assez régulier et flanquées de cinq tours ou bastions demi-cylindrique. Nous savons par ailleurs qu'une basse-cour précédait le corps de place vers le midi.

De prime abord nous ne pouvons nous empêcher d'évoquer le cheflieu des Coucy, érigé vers 1230-1240 à l'extrémité d'un promontoire rocheux et doté d'une singulière puissance. Cette oeuvre-maîtresse de notre architecture militaire associait également un vaste baile à un corps de place dessinant sur le sol un quadrilatère, fort irrégulier cette fois, mais flanqué de quatre tours cylindriques à ses angles. Un énorme donjon circulaire, enveloppé d'une chemise de même forme, se dressait au milieu du front méridional. La porte d'entrée s'ouvrait dans son voisinage, entre la chemise et l'une des tours d'angle ${ }^{4}$. S'autorisant de ces dispositions pour reconstituer sur le papier le défunt château de Saint-Gobain, on a pensé que le cinquième bastion couvrait un donjon, représenté sur les

${ }^{4}$ E. Lefèvre-Pontalis et Ph. Lauer: Le châteal de Coucy, 2. éd., Paris, 1928. 
dessins signalés plus haut, cylindrique lui aussi et auquel on a prêté $23 \mathrm{~m}$. de diamètre à la base. Par malheur on n'a trouvé nulle trace de cet ouvrage dans le sol. L'espace compris entre l'escarpe du bastion et les substructions connues d'une énigmatique bâtisse rectangulaire, proche du milieu de la cour intérieure, ne laisse d'ailleurs pas assez de place pour un donjon aussi volumineux. Le jumelage des bastions implique l'existence d'un ouvrage unique, c'est-à-dire d'un châtelet contenant le porche d'entrée. La porte s'ouvrait assurément entre deux tours dont l'une - celle de droite-_, peut-être plus grosse et plus haute que les autres, aurait en ce cas rempli en outre l'office de donjon ${ }^{5}$.

Revenons à la galerie. C'est un couloir voûté en berceau plein-cintre et large de $2^{\mathrm{m}}, 20$ en général. Selon toute vraisemblance il contournait le corps de place à sa base, en épousant la courbe des bastions. Des archères irrégulièrement distribuées et fort ébrasées, mais réduites aux dimensions de simples fentes ${ }^{6}$, l'éclairaient chichement. On y descendait pas des escaliers assez longs à l'occasion, qui desservaient en outre quelques salles souterraines. D'autres escaliers conduisaient à des poternes débouchant dans les fossés. Les embrasures de tir dénotent le XIII ${ }^{\mathrm{e}}$ siè cle, la première moitié plutôt que la seconde ${ }^{7}$. Il ne saurait s'agir en l'espèce d'une galerie de contremine, car elle dominait d'assez haut le fond des fossés, mais d'une gaine offrant un niveau de tir supplémentaire et s'ajoutant à celui que constituait le chemin de ronde. Les ingénieurs du Moyen Âge ont gratifié çà et là d'organes analogues les forteresses d'Orient et d'Europe ${ }^{8}$.

Je me demande néanmoins pourquoi le constructeur de Saint-Gobain affaiblit les courtines et les bastions du château à brève distance de la base des murs. Je sais bien que la gaine s'adossait au socle rocheux de l'édifice, ce qui eût limité les dégâts causés par l'artillerie. Néanmoins je me sens conduit à supposer que le chemin de ronde n'occupait pas un niveau beaucoup plus élevé et qu'il couronnait tout bonnement ledit socle. Autre anomalie à retenir: le diamètre énorme des bastions, porté à $32 \mathrm{~m}$., donc très supérieur à celui des tours de Coucy — pourtant très grosses en leur temps- qui n'atteignait nulle part $20 \mathrm{~m}$., et sensiblement égal à celui du célèbre donjon d'Enguerrand III, le géant de son temps. J'ai d'abord cru que la place avait été renforcée par le connétable de Saint-Pol, à qui l'on devait le formidable château de Ham en Vermandois

${ }^{5}$ Cette description globale diffère en plusieurs points de celle qu'a énoncée Peychès (op. cit., pp. 35 ss.).

${ }^{6}$ Certaines n'ont que $4 \mathrm{~cm}$. de large.

7 Peychès (op. cit., pp. 21 ss.) a décrit la galerie en détail.

${ }^{8}$ P. Hétıot: Un organe peu connu de la fortification médiévale: la gaine, dans Gladius, X, 1972, pp. 45 ss. 
et son donjon mesurant $33 \mathrm{~m}$. de diamètre ${ }^{9}$. Mais, je le répète, la gaine offre tous les caractères d'une oeuvre du XIII ${ }^{\mathrm{e}}$ siècle, probablement antérieure à 1250 . Force m'est donc de chercher une autre solution: celle d'un château posé dès l'origine sur une plateforme couvrant une surface plus étendue; autrement dit, celle d'un château à deux enceintes concentriques, celle du dehors relativement basse et contournant le sol d'assiette. La longueur des escaliers desservant la gaine nous indique d'ailleurs que le corps de place se dressait en retrait de l'enveloppe extérieure ${ }^{10}$.

En Europe les plus anciennes enceintes concentriques remontaient à l'antiquité préhistorique. Simples levées de terre à l'origine, elles s'effacèrent progressivement, mais sur le tard, devant des ouvrages en pierre. $\mathrm{Au} \mathrm{V}^{\mathrm{e}}$ siècle de notre ère les Byzantins construisirent des spécimens remarquables de la nouvelle manière à Constantinople et à Nicée (Fig. 2). La capitale impériale fût alors environnée de trois lignes de murailles, épousant des tracés parallèles, protégées par un fossé, enfin croissant en hauteur du dehors vers le dedans; la seconde et la dernière seules flanquées de tours ". A Nicée l'on simplifia quelque peu ce dispositif hiérarchisé en réduisant le nombre des ceintures de trois à deux ${ }^{12}$. A vrai dire le dédoublement des remparts - l'un bas en avant du second plus élevéremontait dans le Proche Orient au second millénaire de l'Antiquité. J'en connais des applications déjà savantes, réalisées à cette époque par les Egyptiens en Nubie ${ }^{13}$ et par les Hittites en Anatolie ${ }^{14}$. Ces enceintes successives qu'il fallait forcer l'une après l'autre, n'avaient pas pour unique objet de multiplier les obstacles apportés à l'assaillant. Les lices, c'està-dire le terre-plein annulaire séparant les braies -id est le rempart extérieur- du corps de place, pouvaient recevoir des machines de guerre susceptibles de contenir l'ennemi à distance respectueuse du fossé péri-

9 Cf. A. Mersier: Le château de Ham, dans «Bull. Monumental», LXXVIII, 1914, pp. 232 ss.

${ }^{10}$ Peychès a déjà proposé une restitution analogue à la mienne.

"A. van Millingen: Byzantine Constantinople: the walls of the city..., Londres, 1899, pp. $51-55$ et pl. entre les pp. 106 et 107; Fr. Krischen, B. MeyerPlan't et A. M. Schneider: Die Landmauer von Konstantinopel, Berlin, 19381943.

${ }_{12}$ S. Tox: A bistory of fortification from 3000 b. C. to a. D. 1700 , Londres, 1955, pp. 53-54.

A. W. LaWrence: Ancient Egyptian fortification, dans The Journ. of Egyptian Archaealogy, LI, 1965, pp. 74 ss.; J. VAndiER: Manuel d'archéol. égyptienne, II, 2. ${ }^{\circ}$ partie, Paris, 1955, pp. 997 ss. C'était une variante de la double enceinte des Egyptiens et des Israélites; cf. le P. A.-G. BARrors: Manuel d'archéol. biblique, I, Paris, 1939, pp. 207 ss.

${ }^{14}$ L. Delaporte: Les Hittites, Paris, 1936, p. 279 et fig. de la p. 109; Dr. G. Contenau: La civilisation des Hittites et des Hurrites du Mitanni, Paris, 1948, p. 132. 
phérique. En revanche, envahies par l'adversaire, elles lui fournissaient alors une plateforme d'attaque à pied d'oeuvre. Enfin les braies se prêtaient au tir rasant mieux que les hauts murs.

Les ingénieurs médiévaux ne laissèrent pas de recueillir le principe çà et là. Construit en vertu d'une autorisation que le duc d'Aquitaine

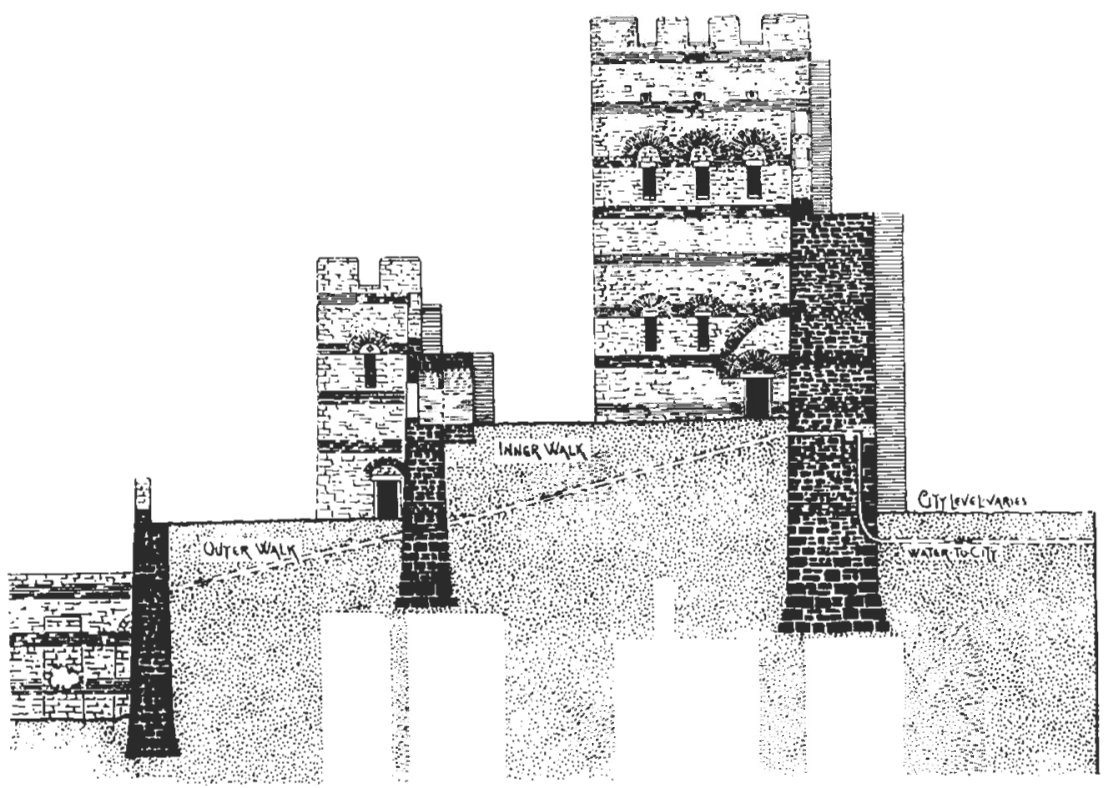

Fig. 2.-Constantinople. Remparts du Bas Empire, coupe restituée par A. E. Henderson.

délivra l'an 1308 à un neveu de Clément V, le château de Budos en Bordelais est un édifice de plan rectangulaire dont les courtines, flanquées de quatre petites tours d'angle, enserrent une cour centrale. Des lices larges de quelques mètres s'interposent entre le corps de place et les fossés. Épousant le tracé des murailles et s'arrondissant en demi-cercle en bordure des tours, elles semblent avoir été dotées d'un mur ou de palissades au dessus de l'escarpe ${ }^{15}$. Ces dispositions reproduisaient tout bonnement celles que je prête à Saint-Gobain. Le château de Morlanne

15 J. Gardelles: Les châteaux du Moyen Âge dans la France du S.-O...., GenèveParis, 1972, p. 109; L. DrouYN: La Guienne militaire..., II, Bordeaux-Paris, 1865, pp. 148 ss. et 155 . 
en Béarn, élevé probablement vers 1370 pour un frère du comte de Foix ${ }^{16}$, avait une enceinte polygonale, dénuée de tours, sauf un donjon. Deux fossés concentriques l'enveloppaient, celui du dedans côtoyant des lices que défendait une palissade ${ }^{17}$. On n'aurait sans doute pas grand'peine à trouver d'autres exemples de cette formule, mais ne retenons désormais que les monuments où l'enceinte extérieure fut entièrement faite en maçonnerie et non en bois.

Allongés et modernisés de 1230 à 1290 ou environ, les remparts de la Cité de Carcassonne furent à cette occasion doublés d'une série continue de braies, flanquées elles aussi de tours ${ }^{18}$. Les plans du XVII ${ }^{c}$ siècle nous montrent autour des murailles de la haute-ville de Boulogne-surMer une ceinture continue de défenses extérieures, alignées sur les fossés et dont un compte de 1415-1416 nous révèle l'existence ${ }^{19}$.

On étendit naturellement le système aux châteaux, avant même que Budos et Morlanne eussent vu le jour, sauf à en varier l'application d'un monument à l'autre pour l'adapter au relief du sol. Dans les forteresses assises en terrain plat les lices et la cour du corps de place occupaient le même niveau, à peu de chose près ${ }^{20}$. Ailleurs elles s'échelonnaient à des altitudes différentes; le corps de place se réservant le sommet de la colline ou de la montagne, tandis que l'enceinte extérieure se développait sur les pentes ${ }^{21}$. En tous cas les remparts du corps de place commandaient invariablement ceux qui les environnaient. Constituée le plus souvent par une muraille assez basse qu'on gratifia au besoin de tours ouvertes à la gorge, l'enceinte extérieure appelle alors le dénomination de braies. Au château de Douvres elle n'est par exception pas moins imposante que celle qu'elle couvre, quoiqu'on l'ait plantée à mi-côte. Ainsi, quelles que fussent les dispositions adoptées, le profil des ouvrages défensifs dessinait un escalier comme à Constantinople.

Le château syrien de Margat (Markab), élevé par les Hospitaliers dans l'ancienne principauté d'Antioche au cours des XII" et XIII ${ }^{e}$ siècles,

${ }^{16}$ R. RitTer: Les constructions militaires de Gaston Fébus en Béarn, Pau, 1923, pp. 47-48.

${ }_{17}$ R. RitTER: Cbâteaux, donjons et places fortes: l'arcbit. militaire française, Paris, 1953 , p. 97.

${ }_{18}$ J. Poux: La Cité de Carcassonne: précis bist, archéol. et descriptif, Toulouse, 1925, pp. 32, 33, 149 ss. et plan à la fin du volume; Y. Bruand: La Cité de Carcassonne: les enceintes fortifiées, dans le Congrès archéol. de France, CXXXI, 1973, pp. 503 ss.

${ }_{19}$ P. HÉliot: Le château de Boulogne-sur-Mer, Paris, 1933, pp. 13 et 43.

${ }^{20}$ Tel était le cas de Margat, Najac, Budos, Blanquefort, Vaujours, de la majorité des châteaux ibériques et britanniques que je vais citer, outre probablement Saint Gobain.

${ }^{21}$ Ainsi de Carcassonne, Boulogne, Chalucet, Bonaguil, Douvres et Peñafiel. 
montrait un excellent exemplaire de la formule avec ses braies renforcées de petites tours et, sur le front le plus exposé, d'un puissant bastion ${ }^{22}$. Celui de Najac en Rouergue, renouvelé sur l'ordre d'Alphonse de Poitiers vers 1253-1260, se dresse sur une plateforme beaucoup plus vaste, environnée d'un rempart bas qui, remontant en partie au XII ${ }^{\mathrm{e}}$ siècle, fut très irrégulièrement tracé ${ }^{23}$. Dans le Haut-Limousin celui de Chalucet, apparemment bâti vers la première moitié du XIII ${ }^{\mathrm{e}}$ siècle, s'assied sur un socle rocheux, émergeant d'une terrasse que délimite une enceinte basse, approximativement rectangulaire et flanquée de quelques tours ${ }^{24}$. Lorsque l'ingénieur Sicard de Lordat, au service de Gaston Fébus, comte de Foix et vicomte de Béarn, modernisa le château de Pau vers 1370. 1378 , il enveloppa le corps de place polygonal, sur plus des trois quarts de son circuit, par des braies assez basses qui remplacèrent sans doute des palissades ${ }^{25}$.

Blanquefort en Bordelais est difficile à dater avec quelque précision, faute de documents écrits suffisamment explicites (Fig. 3). Il y avait là un altier corps de logis rectangulaire en plan, cantonné de six fortes tours; le tout couronné d'une terrasse périphérique qui environnait le dernier étage, élevé en retrait. L'édifice s'implantait sur un terre-plein de forme presque ovale, enveloppé d'une enceinte basse que renforçaient quelques tours et tourelles ${ }^{26}$. Il me semble avoir résulté du développement d'un type de manoirs bâtis dans la région de Bordeaux au cours de la première moitié du XIV siècle ou environ et dont le corps de logis, également rectangulaire, était pourvu de quatre tours ou tourelles d'angle ${ }^{27}$. L'une de ces maisons-fortes, celle du Grand Puch en Bordelais, érigée en vertu d'une autorisation ducale délivrée en 1330 ou 1348, était même circonscrite par des lices larges de plusieurs mètres ${ }^{28}$. Le petit château d'Armentières en Soissonnais, qui remontait apparemment à la fin du siècle précédent, appliquait une solution analogue a celle qui nous occupe en ce sens que les tours, désertant les murs d'enceinte, s'accrochaient également aux angles du corps de logis ${ }^{29}$. En définitive

${ }^{22}$ G. Rey: Étude sur les monum. de l'arcbit. militaire des Croisés en Syrie et dans l'ile de Chypre, Paris, 1871, pp. 21 ss.

${ }^{23}$ Fr. Salet: Najac, dans le Congrès archéol. de France, t. C, 1937, pp. 177 et 180-182; H.-P. Eydoux: Châteaux fantastiques, III, Paris, 1969-1972, pp. 60 ss.

${ }^{24}$ L. Guibert: Cbâlucet, dans le «Bull. de la Soc. Archéol. et Hist. du Limousin», XXXIII, 1886, pp. 119 ss.

${ }^{25}$ R. RitTer: Le cbâteau de Pau, 2." éd., Toulouse-Paris, 1929, pp. 72-74.

${ }^{26}$ Drouyn: op. cit., II, pp. 38 ss. et surtout 56 ss.

${ }^{27}$ Gardelle: op. cit., pp. 76, 77, 83, 144 et fig. 170-177.

${ }^{28}$ Ibíd., p. 164.

${ }^{29}$ Au lieu de se dresser au centre d'un terre-plein, le corps de logis d'Armentières, flanqué de quatre fortes tours, fermait vers le sud une cour que bordait par ailleurs 


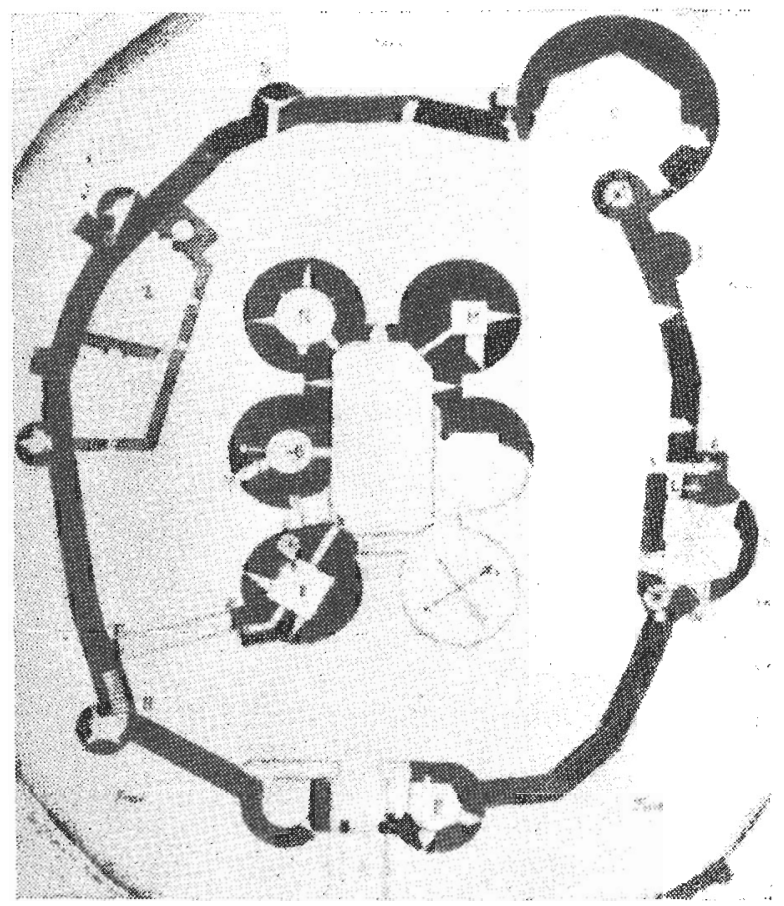

FIG. 3.-Blanquefort. Château, plan d'après L. Drouyn.

Blanquefort fut l'oeuvre d'une période comprise entre la fin du XIII siècle et celle du suivant ${ }^{30}$. Quant à la filiation lointaine de ce type monumental, il convient à mon sens de la chercher, non pas dans les châteaux rectangulaires à tours d'angle et cour centrale, mais dans les don-

une enceinte flanquée de tourelles. Les ressemblances entre ce château et celui de Blanquefort sont surtout sensibles dans les corps de logis, tous deux issus d'une même idée et faisant en quelque mesure figures de donjons. On a sans doute construit Armentières dans la seconde moitié ou à la fin du XIII siècle. Cf. J.-M. Trouvelot: Les château d'Armentières, dans le «Bull. Monumental», LXXXII, 1923, pp. 95 ss.

${ }^{30}$ Drouyn hésitait entre la fin du XIII ${ }^{c}$ siècle et le début du XIV $\mathrm{V}^{*}$, tandis que M. Gardelles (op. cit., pp. 102-103), frappé par certaines ressemblances qui unissaient l'élévation de Blanquefort à celle de la Bastille parisienne, propose une date de construction plus récente et postérieure à 1369. J'avoue qu'aucun des arguments de mes devanciers ne m'ayant convaincu, je préfère renoncer à toute précision et adopter une marge très large. 
jons romans cubiques, cantonnés de quatre tourelles cylindriques, aménagés pour l'habitation et dont la plupart des spécimens connus se trouvent en Poitou, à Niort notamment ${ }^{31}$. Construit vers 1373 , le donjon résidentiel de Nunney en Somerset corrobore mon hypothèse. C'est une bâtisse barlongue dont les quatre tours d'angle ne sont pas plus hautes que les murs droits, comme à Niort et à Blanquefort ${ }^{32}$. Le grossissement des tourelles au gabarit de véritables tours s'était déjà opéré vers 1200 au bénéfice du donjon d'Ambleny en Soissonnais ${ }^{33}$.

Revenons aux enceintes extérieures. La France en a conservé quelques spécimens de l'extrême fin du Moyen Âge, sinon des débuts de la Renaissance, qui offtaient des plateformes à l'usage de l'artillerie à feu. Actuellement en ruines, celui du château de Vaujours en Anjou, tout proche de la Touraine, remonte apparemment au XVe siècle. Le plus connu est à Bonaguil en Agenais, oeuvre d'un seigneur mégalomane bâtie vers 1500 ou peu après. Le château s'enveloppe à l'est, au sud et à l'ouest de larges braies très irrégulièrement tracées, flanquées de tours basses et d'un bastion ${ }^{34}$.

Le thème étudié dans ce mémoire se diffusa dans la Péninsule Ibérique, peut-être dès le milieu ou la seconde moitié du XI $\mathrm{X}^{\mathrm{e}}$ siècle si le château Saint-Georges à Lisbonne remonte bien à cette époque ${ }^{35}$, mais il y offrit d'intéressantes variantes. Deux grands châteaux de la province de Tolède, apparemment renouvelés au XIV $\mathrm{X}^{e}$ siècle, nous montrent de hautes courtines, précédées de braies sur leurs fronts les plus exposés, mais, par exception, les tours de l'enceinte intéricure y sont assez proéminentes pour enjamber les lices, qui passent sous de hautes arches, et faire saillie sur les braies; procédé inventé en Orient dès l'Antiquité. Ainsi

31 Cf, A. Chîtelain: Domions romans des pays d'Ouest, Paris, 1973, pp. 34-35; P. HÉliot: Les donjons de Niort et la fortification médiévale, dans la «Rev. du Bas-Poitou et des Provinces de l'Ouest», 1970, pp. 45 ss. et notamment 63 ss.

${ }_{32}$ R. A. Brown: English medieval castles, Londres, 1954, p. 93; S. Toy: The caslles of Great Britain, 2." éd., Londres, 1954, p. 222.

${ }_{33}$ P. Hélior: L'évolution des donjons dans le N.O. de la France et l'Angleterre au XII siècle, dans le «Bull. Archéol. du Comité des Travaux Hist.», nouv. série, V, 1969 , p. 166.

${ }^{34}$ M. Pons: Regards sur Bonaguil, St.-Front-s/Lémance, 1971, pp. 20 et 40 ss.; Eydoux: op. cit., I, pp. 98, 102 et 104; chan. Marboutin: Bonaguil, dans le Congrès, cit., de 1937, pp. 307 et 314 ss.; PH. Lnuzun: Le châteall de Bonaguil en Agenais, 2." éd., Paris-Agen, 1884, pp. $36-41$ et 47-49. M. M. Pons a publié des images suggestives dans son livre sur Bonaguil, château de rêve, Toulouse, 1959, phot. de la couverture et en face de la p. 97. M. J. Gardelle a étudié Le premier château de Boneguil, dans le Congrès, cit., CXXVII, 1969, pp. 206 ss.

${ }^{35} \mathrm{O}$ castelo de S. Jorge, dans le «Boletim da Direç̧ão Geral dos Edifícios e Monum. Nacionais», XXV-XXVI, 1941; N. DE ARaújo: Inventario de Lisboa, I, Lisbonne, 1944, pp. 13 et 16. 
d'Escalona ${ }^{36}$ et de Montalbán, celui-ci réédifié par les Coronel vers 1323 , en tous cas entre 1308 et $1353^{37}$. A Muñatones en Biscaye le donjon central s'enveloppe de hautes courtines, dépourvues de tout organe de flanquement, et de braies que renforcent de petites tours ${ }^{33}$. C'est encore une oeuvre du XIV siècle. Au cours du XV $\mathrm{XV}^{\mathrm{e}}$ la formule suscita en Castille plusieurs applications très remarquables, caractérisées par un plan rectangulaire plus ou mons régulier. Ainsi de Coca près de Ségovie ${ }^{39}$, de la Mota à Medina del Campo (Fig. 4) ${ }^{40}$, de Manzanares el Real dans la province de Madrid ${ }^{41}$ et de Guadamur dans le secteur tolédan ${ }^{42}$. Le curieux château de Peñafiel dans la région de Valladolid chevauche une longue arête rocheuse, qui lui impose une singulière étroitesse ${ }^{43}$.

À l'exception du premier et des deux derniers les spécimens britanniques forment un groupe homogène, qu'expliquent leur contemporanéité autant que les circonstances de leur création. Le doyen d'âge n'est autre que le château royal de Douvres où l'enceinte extérieure, plus puissante encore que celle qui couronne la colline, n'offre dans son tracé aucun parallélisme avec elle. Ses hautes courtines et ses nombreuses tours furent bâties de la fin du XII ${ }^{\mathrm{e}}$ siècle au milieu du XIII ${ }^{44}$. L'idée fit son chemin outre-Manche puisqu'on l'appliqua derechef à la Tour, c'està-dire à la citadelle de Londres. Ici pourtant l'horizontalité du terrain

${ }^{36}$ F. Chueca Gortia: Historia de la arquitectura española: Edad Antigua y Edad Media, Madrid, 1965, p. 534; V. Lampérez y Romea: Arquitectura civil española de los siglos I al XVIII, I, Madrid, 1922, pp. 272 ss.; F. B. Nayarro: Fortalezas y castillos de Maqueda y Escalona, dans le «Boletín de la Soc. Española de Excursiones», III, 1895, fig, des pp. 29 et 30; Gladius, IV, 1965, fig. des p. 99 Sur la date de l'enceinte L. Torres Balbás dans Ars Hispaniae, IV, Madrid, 1949, pp. 324 et 341 .

${ }^{37}$ Chueca Goitia, loc. cit.; Torres Balbás: op. cit., pp. 341-342; ck de CeDILLO: El castillo de Montalbán, dans le «Boletín...», cit., XXXII, 1924, pp. 141145.

${ }^{36}$ Chueca Gortin: op. cit., p. 655. Le château catalan de Bellcaire (fin du $\mathrm{XIII}^{e}$ siècle) n'appartient pas à la même famille monumentale quoique son plan nous le laisse supposer. En réalité il n'y a qu'une seule enceinte, englobant un bloc de bâtiments résidentiels. Voir ibid., p. 643; L. Monreal et M. DE Riquer: Els castells medievals de Catalunya, Barcelone, 1955, pp. 113 et 115 ss.

${ }^{39}$ Chueca Goitia: op. cit., p. 537.

40 Ibid., pp. 535-536; Y. Brund: De l'importance bist. et de la valeur militaire des ouvrages fortifiés en Vieille-Castille au XV' siècle, dans Le Moyen Äge, 1957, pp. 79-80; A. Fernández Casanova: El castillo de la Mota, dans le «Bulletin...», cit., XII, 1904, p. 10.

"41 Chueca Goitia: op. cit., pp. 670-671.

${ }^{42}$ Lampérez y Romea: op. cit., I, p. 272; C. Sarthou Carreras: Castillos de España, 4." éd., Madrid, 1969, fig. des p. 256 et 257.

${ }^{43}$ Chueca Goitia: op. cit., p. 648; Bruand: op - cit., pp. 77-78; Sarthou CaRRERAS: op. cit., fig. des pp. 383 ss.

${ }^{4}$ R. A. Brown: Dover castle, Londres, 1966, pp. 6-9 et 12 ss. 


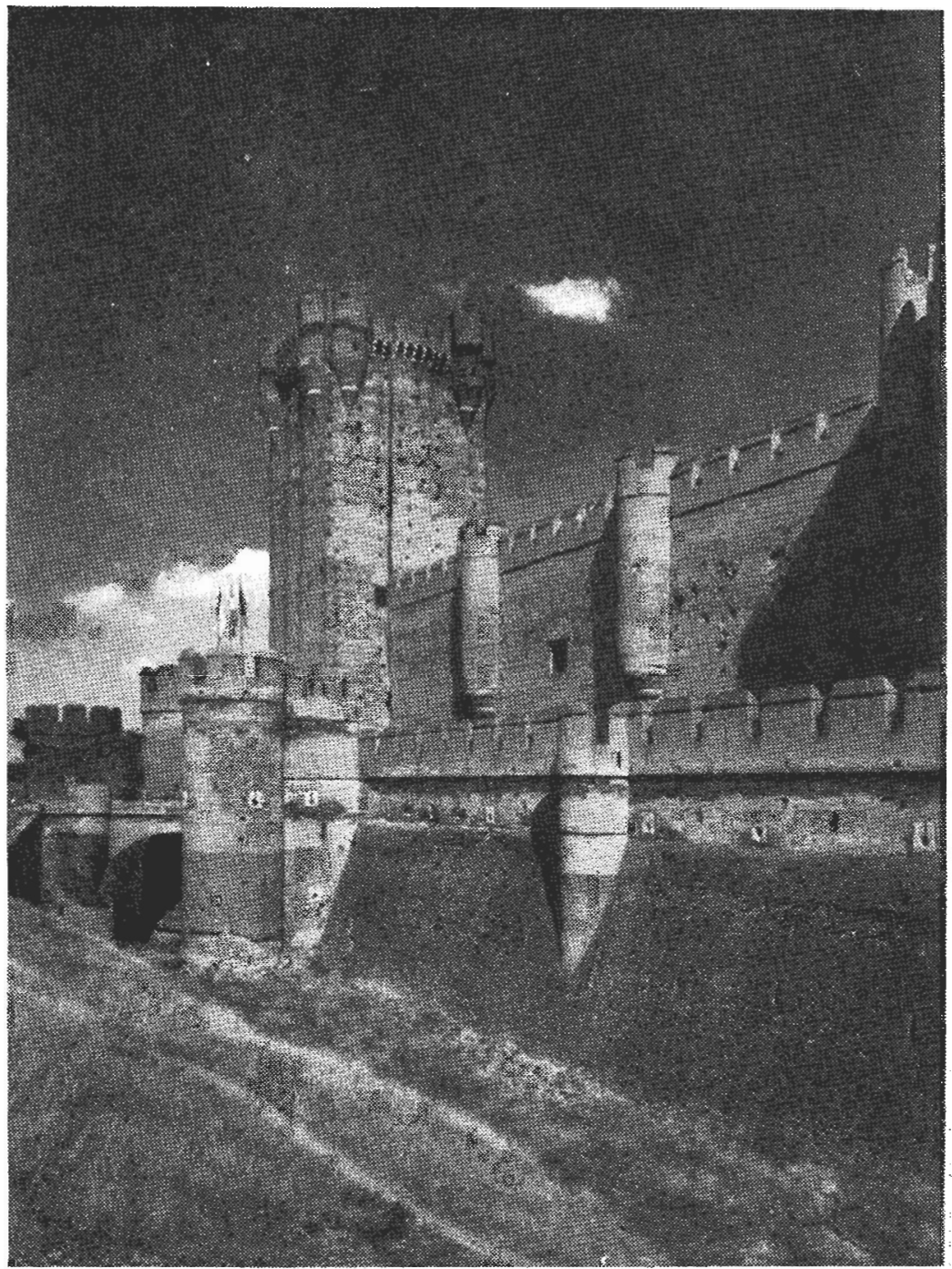

Fic. 4.-Châtcau espagnol de La Mota. Medina del Campo, prov. Valladolid. $\mathrm{xv}$ siècle. 
a permis aux braies, ajoutées de 1275 à 1285 , de suivre le tracé polygonal du corps de place ${ }^{45}$. A la même époque, c'est-à-dire de 1217 à 1280 environ, un comte d'Hertford et Gloucester fit construire à Caerphilly, dans le Glamorgan, un château rectangulaire, assis sur une plateforme de même plan que borde une enceinte basse ${ }^{45}$. Quelques années plus tard Edouard $I^{\text {er }}$ faisait appliquer la formule, sous la direction de son meilleur ingénieur - Jacques de Saint-Georges-, à plusieurs de ses châteaux du pays de Galles: Rhuddlan au comté de Flint de 1277 à $1282^{47}$, Harlech en Merioneth de 1283 à $1290^{48}$, enfin Beaumaris dans l'île d'Anglesey, commencé l'an 1295, achevé dans son gros oeuvre en $1330^{49}$. Les braies d'Harlech (Fig. 5) et de Beaumaris épousent le tracé du corps de place et sont flanquées de tours. Les lices qu'elles circonscrivent occupent, à peu de chose près, le même niveau que la cour intérieure à l'exemple de Londres et de Caerphilly.

On appliqua le principe jusqu'au château de Raglan dans le comté de Monmouth, sauf à le limiter au donjon hexagonal, planté sur une ancienne motte, au dehors du corps de place. Vers 1450-1469 on affermit en effet la motte en la corsetant d'une chemise basse à six faces, dotée de tourelles d'angle, et en ménageant des lices entre le mur de soutènement et la grosse tour ${ }^{50}$.

Le plus curieux des examplaires britanniques se trouvait jadis à Queenborough dans le Kent (Fig. 6). Bâti sur l'ordre d'Edouard III de 1361 à 1375, sans doute sous la direction de l'architecte John Box, il remplissait la double mission de résidence royale et de poste de surveillance sur les rives méridionales de l'estuaire de la Tamise. Rasé au XVII" siècle, il se laisse quand même restituer dans ses dispositions générales. C'était un château de plan concentrique et rayonnant à la fois. Rigou-

${ }^{45}$ Royal Commission on bist. monum. (England): an inventory of the bist. monum. in London, V, Londres, 1930, pp. 76 ss.; H. M. Colvin, R. A. Brown et A. J. 'TAYlor: The bistory of the King's works: the Middle Ages, Londres, 1963, pp. 715 ss.; BRown: English medieval castles, op. cit., p. 83.

${ }^{46}$ Caerphilly castle, Londres, 1959 (guide officiel du Ministry of Works); Toy: Castles of Great Britain, op. cit., pp. 164-166; Brown: op. cit., p. 79.

${ }^{47}$ A. J. Taylor: Rhuddlan castle, Londres, 1956; Colvin, Brown et Taylor: op. cit., pp. 378 ss.

${ }^{48}$ Harlech castle, Londres, 1966 (guide officiel du Ministry of Works): Colvin, Brown et TAYLOR: op. cil., pp. 357 ss.; Toy: op. cit., pp. 170-173. Voir aussi les figures dans A. Phillips: Harlech caslle, Londres, 1965.

"Royal Commission on ancient and bist. monum. in Wales and Monmouthshire: an inventory of the ancient monum. in Anglesey, Londres, 1937, pp. 8 ss.; Colvin, Brown et TAYLOR: op. cit., pp. 395 ss.; TOY: op. cit., pp. 173-175; Brown: op. cit.. p. 76.

so A. J. TAylor: Raglan castle, Londres, 1950, pp. 24-25, $41-42$ et pl. 1, y BROWN: op. cit., pp. $92-93$ et 98 (Fig. 51). 
reusement circulaire et dotée seulement de deux tours jumelles qui encadraient l'entrée, l'enceinte extérieure enveloppait de larges lices sous la protection d'un fossé. Au milieu de cette plateforme s'élevait le corps de place, dont les murailles décrivaient également un cercle complet. Six tours régulièrement espacées flanquaient des courtines au revers desquelles s'adossait une ceinture de bâtiments. Les murs qui compartimen-

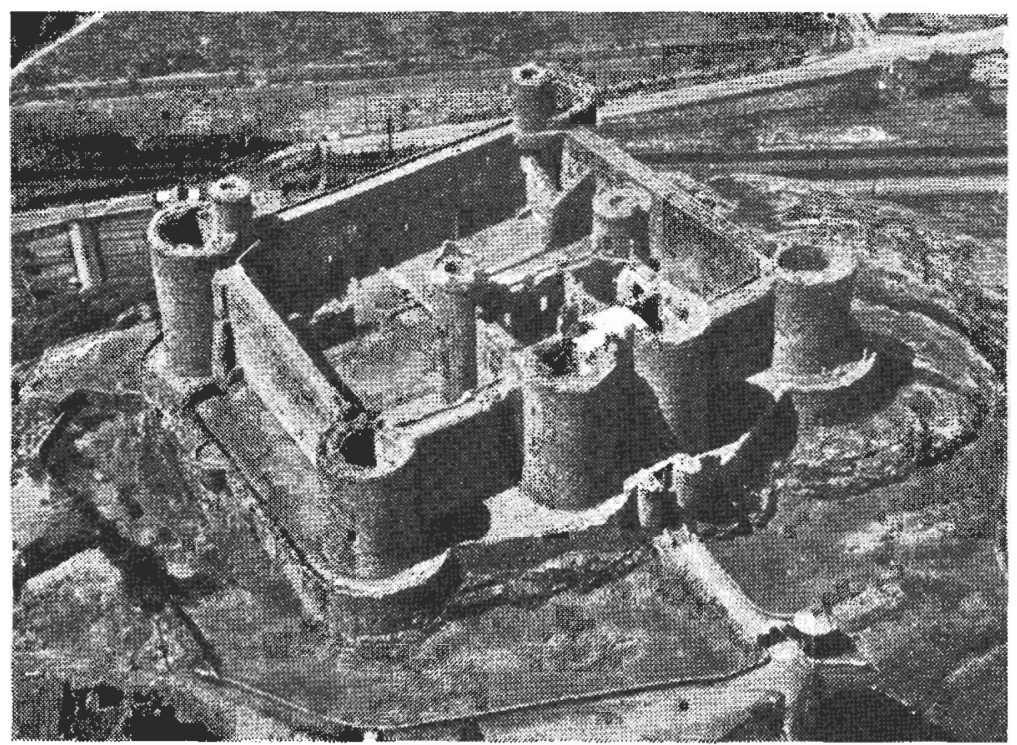

Fig. 5.-Harlech. Château, vue aérienne (Copyright Aerofilms and Aero Pictorial Ltd.).

taient les corps de logis convergeaient à peu près vers le milieu de la cour centrale ${ }^{51}$. Ce plan radio-concentrique quasi parfait, unique en Angleterre, devait être repris durant le XVI ${ }^{\circ}$ siècle, mutatis mutandis, au profit des forts côtiers érigés sous Henri VIII ${ }^{52}$.

Reste à savoir quelle en fut la genèse. Pour les braies la chose semble claire, car il suffisait de se reporter aux précédents britanniques que je viens d'énumérer, sauf à leur imposer un tracé différent. En revanche le

"1 Colvin, Brown y Thyior: op. cit., pp. 793 ss.; Brown, op. cit., pp. 93-94.

52 B. H. ST. I. O’Neil: Castles and cannon..., Oxford, 1960, pp. 53 ss. 
château proprement dit pose un problème de filiation difficile à résoudre. Faut-il l'expliquer par un développement de quelques shell-keeps romans d'outre-Manche, construits sur des mottes et tels qu'à Restormel en Cornouaille? L'enceinte circulaire de ce dernier était à l'origine flanquée d'une tour. Des bâtiments s'y adossaient, enserrant une cour centrale et divisés par des murs de refend approximativement rayonnants ${ }^{53}$. L'hypo-

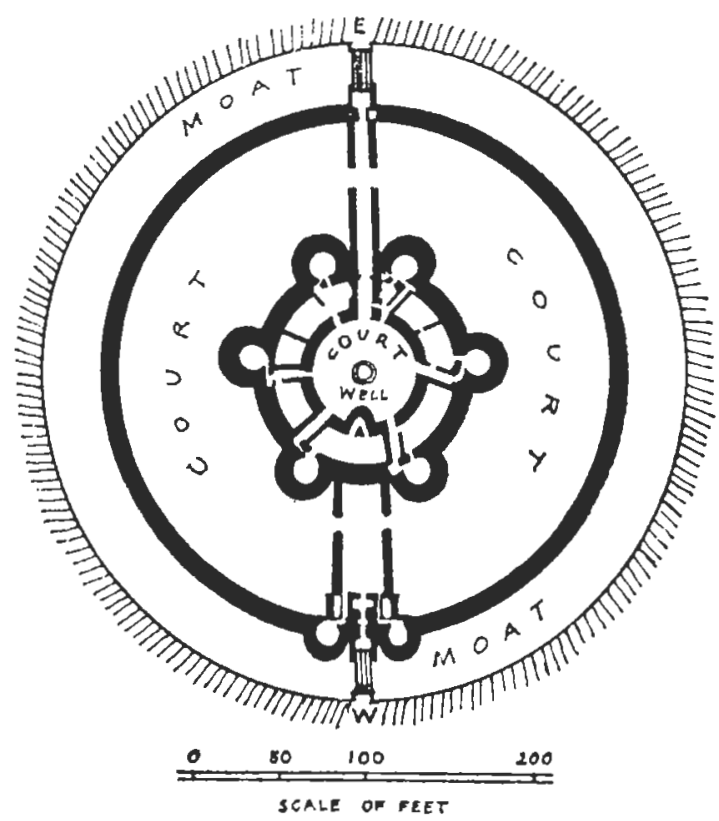

Fig. 6.-Queenborough. Château, plan d'après Sir A. W. Clapham.

thèse est plausible quoique je ne connaisse aucun intermédiaire entre ces modèles supposés, antérieurs au XIII ${ }^{\mathrm{e}}$ siècle, et la forteresse très savamment conçue d'Edouard III.

Pourquoi ne pas chercher les sources d'inspiration dans le Midi? Le château royal de Bellver dans l'île de Majorque me vient tout de suite à l'esprit à cause de son plan circulaire, de ses courtines et de ses bâtiments enveloppant une cour centrale, à cause des trois tours et des quatre tourelles affirmant son caractère militaire. En contrepartie il y

${ }_{53}$ ToY: op. cit., pp. 62-63; Brown: op. cit., p. 39 (Fig, 14). 
existe un donjon planté à l'écart, tandis que les lices y font défaut ${ }^{54}$. L'édifice est également exceptionnel en raison de sa forme, mais ressortit pour le reste à la tradition méditerranéenne des palais fortifiés. Il jouit en outre du privilège de la priorité puisqu'il vit le jour vers 1300 1310. D'autres sources sont cependant possibles. La rigoureuse symétrie de Queenborough dénote en effet une conception profondément classique de l'architecture profane, qu'on ne trouvait pas au même degré dans l'Europe médiévale, si ce n'est en quelques monuments italiens. Me voici conduit à introduire dans le débat le Castel del Monte, construit par l'empereur Frédéric II en Apulie vers 1240-1250. À part le tracé octogonal des murs d'enveloppe et des tours, des bâtiments et de la cour médiane, les ressemblances avec la forteresse d'Edouard III sont aveuglantes, et l'on reconnaît sur la résidence impériale quelques uns des traits caractéristiques essentiels du palais fortifié riverain de la Tamise, à commencer par le plan radioconcentrique régulier ${ }^{55}$. En définitive l'architecte de Queenborough a peut-être combiné deux formules complètement étrangères l'une à l'autre, quoique offrant des dispositions similaires; plus précisément avoir modernisé celle du shell-keep indigène, apparemment tombée depuis longtemps en désuétude, sous l'influence de palais fortifiés du Midi méditerranéen, tels que Bellver et Castel del Monte.

L'étude des vestiges retrouvés et les comparaisons que je viens de faire m'autorisent à me représenter le château de Saint-Gobain de la façon suivante. A la base un piédestal naturel, coupé de son environnement par un fossé profond, circonscrit par une enceinte relativement basse et flanqué de cinq gros bastions. Sur ce socle se dressait en retrait le corps de place qui se pliait au tracé des remparts extérieurs. Les courtines et les cinq tours, dont quatre aux angles, semblaient jaillir des lices. L'entrée s'ouvrait entre la tour de l'angle sud-ouest et celle, toute voisine, qui regardait le midi. Les bastions de grand diamètre qui portaient ces tours devaient se rééditer à la fin du XIII' siècle et au XV ${ }^{c}$ à Caerphilly, Harlech et Manzanares el Real. On retrouve le souvenir du thème jusque sur une résidence aristocratique des ultimes décennies du Moyen Âge: le château du Plessis-Bourré, construit en plein Anjou par l'un des hauts fonctionnaires de Louis XI de 1468 à 1473, et dont l'appareil défensif est actuellement presque inexistant. Ses quatre corps de bâtiment et ses quatre tours d'angle enserrent une cour centrale rectan-

5. M. Durliat: L'art dans le royaume de Majorque, Toulouse, 1962, pp. 236 ss. et 244 ss.

${ }_{55}$ L. Bruenns: Hobenstautenschlösser in Deutschland und Italien, Koenigstein, 1959, pp. 14 ss. 
gulaire et surgissent d'une sorte de socle bas, épousant le tracé de l'édifice à un niveau à peine supérieur à celui du plan d'eau des douves ${ }^{56}$. Je me demande néanmoins si, dans leur état premier, les lices n'étaient pas bordées de braies véritables au lieu du simple parapet que nous voyons aujourd'hui.

En définitive, Saint-Gobain comptait parmi les plus anciens exemplaires connus d'une famille de châteaux d'Occident, dont je viens de passer plusieurs membres en revue. Ces monuments sont caractérisés par leurs lignes de défense concentriques, échelonnées en hauteur de telle manière que l'enceinte intérieure commande l'autre avec une autorité frappante. Une hiérarchisation marquée à ce point ne saurait trouver ses références dans les oeuvres simplistes de la préhistoire européenne. C'est un legs des savantes forteresses méditerranéennes, probablement importé d'Orient à la faveur des Croisades. Si perfectionné qu'il fût, en revanche fort onéreux pour les propriétaires, le système ne parvint pas à supplanter la tradition nordique des ouvrages extérieurs en terre et en bois, qu'on perpétua couramment, en France du moins, jusqu'au delà du Moyen Âge. Il fut peut-être moins exceptionnel autrefois dans l'Europe méridionale, plus proche des sources probables, que dans les pays continentaux du Nord.

56 Fr. Gébelin: Les châteaux de la Loire, Paris, 1931, pp. 33 ss. L'auteur considère l'existence ancienne des braies comme certaine. Sur l'édifice voir surtout P.-M. Auzas: Le château du Plessis-Bourré, dans le Congrès archéol. de France, CXXII, 1964, pp. 252 ss., quoique les lices y soient tout bonnement qualifiées de «promenoir». 